\author{
Associate Professor Xing Yu, PhD \\ E-mail: yuxing@mail.ccnu.edu.cn \\ School of Economics and Business Administration \\ Central China Normal University \\ Professor Weiguo Zhang, PhD \\ E-mail: wgzhang@mail.scut.edu.cn \\ Professor Yongjun Liu, PhD \\ E-mail: bmyjliu@mail.scut.edu.cn \\ School of Business Administration, South China University of \\ Technology
}

\section{CRUDE OIL OPTIONS HEDGING BASED ON A NEW EXTREME RISK MEASURE}

Abstract. This paper investigates the problem of the risk management for an import firm by using crude oil options. We propose a new risk measure (thereafter called CVaRMD) that synthesizes the mean and median deviation of the hedged portfolio loss to trace the extreme risk. The classical volatility models (GARCH-n, GARCH-t, GJR-n and GJR-t) are commonly-used in finance literature to depict the marginal distributions of the oil price and the exchange rate. Different from the time-consuming method of simulation, we employ Copula functions to deduce the cumulative distribution function of the hedged portfolio and then to calculate the extreme risk. Empirical studies demonstrate that GARCH-n and GARCH-t models are better to forecast the volatilities of Brent crude oil price and the exchange rate (CNY/USD)respectively. Frank copula better portrays the correlation structure between Brent crude oil price and the exchange rate. We find that hedging with crude oil options can reduce the extreme risk effectively. By comparing the skewness of the extreme loss under two risk measures of Conditional value-at-risk $(C V a R)$ and CVaRMD, we find that the left deviation degree of the extreme loss under CVaRMD criterion is greater. The result of variance analysis further confirms this conclusion. That is to say, based on the strategy of minimizing CVaRMD, the extreme loss risk that the firm faces is relatively smaller. We further analyze the parameter sensitivities and give the firm some suggestions to choose the appropriate option contracts and decide its budget.

Keywords: risk management, hedging with options, Conditional Value-at-Risk, CVaRMD, Copula function.

\section{JEL Classification: G13, G15, G32}

\section{Introduction}

Crude oil price and exchange rate (especially the exchange rate with respect to USD) risk are prevail for an import or export firm. How to hedge these two risks is an important issue to be addressed by the firm. Intuition tells us to hedge against 
Xing Yu, Weiguo Zhang, Yongjun Liu

major risk first. However, which risk is the major one is a controversial issue. To identify the mutual conduction mechanism between the crude oil price and the exchange rate, researchers have presented many econometric models to discover the source of risk. By using a copula approach and the DCC-MGARCH model, Brayek et al.(2015) examined the relationship between oil prices and the US dollar exchange rate. Rivieccio and Luca (2016) applied a vine copula approach to investigate the dynamic relationship between energy, stock and currency markets. Through their studies, some researchers find that the variability of exchange rates could spill over to the international crude oil market (see e.g. Salisu (2013) and Brahmasrene et al.(2015)). A reason is that USD is the major invoicing currency for international crude oil trading. And crude oils are commonly priced in US dollars. Therefore, fluctuations of oil prices highly depend on the dollar's exchange rate. Referring to other currencies, Ghosh (2011) employed GARCH and EGARCH models to examine the impact of oil price shocks on nominal exchange rate. Ito et al. (2013) investigated the relationship among Japanese firms'risk exposure of the exchange rate and found that, firms with greater dependency on sales in foreign markets had greater foreign exchange exposure. The discussions mentioned above imply that the exchange rate is a critical factor in the international trade. On the other hand, some researchers claimed that exchange rates are affected by crude oil prices. Chen et al. (2016) found that oil price shocks played more important role in dollar exchange rate variations. From different views of the relation between the crude oil price and the exchange rate mentioned above, we conclude that the crude oil price is interacted with the exchange rate. Neither commodity price nor exchange rate risk can be ignored. Thus, in this paper we consider the dependence between the crude oil price and the exchange rate.

Many scholars have investigated the theoretical or empirical hedging motivations to avoid risks of commodity price and exchange rate. They conclude that the firms can basically opt linear (e.g. futures, forwards) or non-linear (e.g. options) instruments to hedge risk. One important strand of the existing literatures is related to the behavior of a competitive firm when futures market exists for hedging purposes. Two notable results of Separation Theorem and Full-hedging Theorem emerge. By taking a comparison, some other researchers believe that futures is a better hedging instrument than option. As we know, futures is a linear hedging instrument and it is suitable to be used to hedge linear risk. While nonlinear structure of option is designed to eliminate extreme the downside risk. Thus, options are proven to be useful to hedge the nonlinear risk because of their nonlinear profits. Wong (2009) demonstrated that the hedging role of currency options was due to two distinct sources of non-linearity including the multiplicative nature of the price and exchange rate and the marginal utility function of the firm. Moreover, relative to futures hedging, buying options for hedging has an advantage in avoiding market-to-market risk reflected as the liquidity risk. According to Committee on Payments and Settlement Systems, firms should take liquidity risk seriously when devising their risk management strategies. 


\section{Crude Oil Options Hedging Based on a New Extreme Risk Measure}

Either buying or selling futures for hedging, the firm is inevitably confront with the funding liquidity risk. However, the market-to-market risk absents when the firm only purchases options to hedge the risk exposure. Therefore, in this paper we assume that there is no currency option market while the crude oil option market is available for hedging oil price risk.

To better describe the investment risk, a variety of risk measures are applied in finance. In the classical Markowitz model, risk is measured by variance or standard deviation. Due to the asymmetric risk in financial practice, the downside risk measure of Value-at-Risk (VaR) is widely used by financial institutions. By minimizing VaR, Ahn et al. (1999) presented an analytical solution of the option hedging problem for an institution. But, VaR is not a coherent risk measure and without subadditivity and convexity. To overcome the defects of VaR, conditional value at risk (CVaR) has arisen. CVaR (also known as expected shortfall (ES) or expected tail loss) is a coherent and spectral measure of risk. Then, CVaR is widely used as the objective function in portfolio theory. Melnikov and Smirnov (2012) studied the problem of partial hedging by constructing hedging strategies that minimized CVaR of the portfolio. However, CVaR portrays the mean of the extreme loss, it cannot reflect the extreme situation. With regard to the extreme risk, Wong and Ming (2009) investigated a new tool called median shortfall to measure risk, wherein the objective function was to minimize the median shortfall. Nevertheless, only using CVaR or median to measure the extreme risk has some deficiencies. For example, when CVaR or median of the loss are identical upon different strategies, evidence on CVaR seems powerless to decide which strategy is better. To overcome the deficiencies of the existing risk measure, we propose a new risk measure, which is the sum of CVaR and the median deviation (or MD for short), wherein MD is the first-order absolute moment of the shortfall loss median. The new risk measure is thereafter called CVaRMD. Since the mean and extreme loss are taken account, the main advantage of the proposed risk measure synthesizes both the overall and individual extreme risk.

To estimate the risk of the hedged portfolio accurately, it is necessary to capture the non-linear inter-dependence of the asset returns. Copula functions are applied to describe the inter-dependence among innovations. Chen and $\mathrm{Tu}$ (2013) estimated the hedged portfolio's VaR using the conditional copula. Zhang et al. (2013) used Monte Carlo method to forecast VaR and ES of the international stock markets portfolio. Domino and Blachowicz (2015) used copula functions for modeling the risk of investment in shares traded on world stock exchanges. Rivieccio and Luca (2016) derived the classical and copula-based VaR conditional expected returns and covariance. Referring to hedging with options, Bajo and Romagnoli (2015) modeled the dependence structure through a copula function. They developed a theoretical hedging model with options. However, a defect of the theoretical model is the assumption of log-normal dynamics for both assets and quantity. It is a stylized fact that financial returns do not show any normal behavior. 
Xing Yu, Weiguo Zhang, Yongjun Liu

GARCH models have become important in time series analysis, particularly in financial applications to analyze and forecast volatility. Deng et al. (2011) applied pair Copula to capture the inter-dependence structure between assets and constructed pair Copula-GARCH-EVT model to minimize CVaR. Dinicặ and Balea (2014) analyzed the evolution of U.S. natural gas spot and futures prices and estimated static and time varying optimal hedge ratios (OHR) through OLS method with different rolling window lengths and bivariate GARCH model with ECM errors (B-GARCH). Since the mean loss is obtained by the weighted method and ignores the extreme individual circumstances, CVaR cannot distinguish the better investment strategy when the values of CVaR are identical under different strategies. To our knowledge, among those studies that have considered options hedging, few aim to the extreme loss problem. The main contributions of this paper are as follows. We propose a new risk measure to cover with the tail extreme risk. The new risk measure called CVaRMD is a compound of the mean and the median deviation of the extreme loss. Furthermore, the existing simulation method of solving the option hedging models is generally time-consuming. By modelling the dependence structure through a copula function, we express the multivariate distribution function as a one dimension integral form. Based on this integration, we calculate the values of different risk measures with an easier routine.

The remainder of this paper is structured as follows. Section 2 proposes a new risk measure called CVaRMD and then discusses the properties of the proposed risk measure. It gives the copula based representation of the hedged portfolio's distribution function and presents the computational procedure based on CopulaGARCH method. Section 3 provides an empirical study to illustrate the ideas of oil options hedging model and shows the advantages of CVaRMD over CVaR with regard to reducing the extreme risk. Variance analysis is used to verify the superiority of the risk measure CVaRMD. Furthermore, we provide some risk management suggestions for the oil import firm. Finally, conclusions and potentials for future research are discussed in Section 4.

\section{The hedging models with options}

In this section, we first describe the call options hedging problem faced by an import firm. Then, we introduce a new risk measure, which can trace the extreme risk well. With the aim to minimize the extreme risk of the hedged portfolio, the optimal options hedging model is then presented.

We assume that a firm currently (at time 0 ) expects to import a quantity $Q$ of the commodities that have a known current price $P_{0}$. The current spot exchange rate, expressed in units of home currency per the foreign currency is $S_{0}$. Let $P_{T}$ and $S_{T}$ be the uncertain commodity price and the exchange rate at a future time $T$. $p_{0}$ denotes the premium of a call option written on $P_{T}$ struck at $K_{p}$. Constraint on the hedging cost is motivated by practicality and liquidity. And the firm is supposed to hedge the naked position by buying amount of $Z_{p}$ call options given the cost constraint of $C$. Since Ahn et al. (1999) pointed out that it was rational to fully spend the budget 


\section{Crude Oil Options Hedging Based on a New Extreme Risk Measure}

for a better hedge effect, we let $C=S_{0} Z_{p}, C=S_{0} Z_{p}$. Factors of IAS or other hedge accounting standards are assumed to be ignored. Under the assumptions mentioned above, when the firm buys commodity options for hedging, then the gain at maturity on the hedged portfolio is written by

$$
\Pi_{T}=P_{0} S_{0}-P_{T} S_{T}+\left[\left(P_{T}-K_{p}\right)^{+}-p_{0}\right] S_{T} Z_{p}
$$

where $Z_{p}$ is the position of the commodity options.

\subsection{A new risk measure CVaRMD}

The main objective in risk management is to evaluate and improve the performance of financial organizations in light of taken the risk. The common used risk measure is VaR. It is the maximum potential loss that a financial asset can suffer with a certainty probability during a certain holding period. For any $\alpha \in$ $(0,1), \mathrm{VaR}$ of $\Pi_{T}$ at a given confidence level of $1-\alpha$ is defined by $\operatorname{VaR}^{\alpha}\left(\Pi_{T}\right)=$ $-q^{\alpha}\left(\Pi_{T}\right)$, where $q^{\alpha}\left(\Pi_{T}\right)$ is the $\alpha$-quantile of $\Pi_{T}$. However, VaR is criticized since it cannot capture the extreme risk. Then, $\mathrm{CVaR}$ was proposed to overcome the deficiencies of $\mathrm{VaR}$. The definition of $\mathrm{CVaR}$ corresponding to $\Pi_{T}$ is

$$
\operatorname{CVaR}^{\alpha}\left(\Pi_{T}\right)=E\left[\Pi_{T} \mid \Pi_{T} \leq q^{\alpha}\left(\Pi_{T}\right)\right] .
$$

As seen in Eq.(2), CVaR is the mean of the loss that more than VaR. Although $\mathrm{CVaR}$ is believed to be superior to VaR in measuring financial risk and has been widely used in risk measure in recent years, it has two intuitive deficiencies. As we know, in the management of portfolio or hedging, investor generally decide the optimal strategies based on some risk control criteria, such as to minimize the risk of VaR or CVaR. We can insight into one deficiency of CVaR is that, when the values of CVaR upon different strategies are identical, investors cannot distinguish which strategy is better. Besides, since CVaR measures the mean loss, it does not take into account the potential individual extreme loss. We explain the aforementioned deficiencies of $\mathrm{CVaR}$ through an example.

Example. Suppose that there are four different candidate investment strategies for the firm to choose. For simplicity, we suppose that every loss occurs with an equal probability of 0.2 . Each row of Table 1 represents a strategy:

Table 1. Strategies and loss

\begin{tabular}{l|lllll|l|lllll}
\hline Strategy & \multicolumn{3}{|l|}{ Loss that larger than } & Strategy & \multicolumn{5}{l}{ Loss that larger than VaR } \\
& VaR & & & & & & & & \\
\hline Strategy.1 & 10 & 8 & 4 & 2 & 1 & Strategy.3 & 226 & 225 & 224 & 223 & 222 \\
Strategy.2 & 7 & 6 & 5 & 4 & 3 & Strategy.4 & 300 & 225 & 224 & 223 & 222 \\
\hline
\end{tabular}

Take strategy 1 as an example, the value of CVaR is -5 , equally to the mean of $10,-8,-4,-2$ and -1 . Respectively, CVaR of strategies $2-4$ are $-5,-24,-238.8$. If the risk evaluation criteria of the strategies are CVaR, then Strategies 1 and 2 are no distinction. That is to say, based on the criteria of CVaR, we can't distinguish which 
Xing Yu, Weiguo Zhang, Yongjun Liu

one is better between Strategy 1 and Strategy 2. In the aspect of the individual extreme loss, the largest loss in Strategies 1 is 10 and 7 in Strategies 2. Then, we can speculate that Strategy 1 is better than Strategy 2. While, how to describe the individual extreme loss quantitatively is another issue that needs to be addressed. We further examine a new criteria of median deviation, abbreviated MD. Here, MD is the expected median deviation of the extreme loss, wherein the median of Strategies 1-4 are $-4,-5,-224$ and -224. And the values of MD in the four Strategies are 3, 1.2, 1.2 and 2. We can find that the values of CVaR in Strategy 1-2, median in Strategy 3-4 and MD in Strategy 2-3 are respectively equal to each other. It is inferred that a separate criteria of CVaR, median or MD does not distinguish well among the strategies. To this end, we propose a new risk measure called CVaRMD (Marked with positive numbers), which is a combination of CVaR and $\mathrm{MD}$. One reason for this consideration is that CVaR and MD can be used to measure the mean and the individual extreme risk. The values of CVaRMD in the four strategies are 8, 6.5, 225.2 and 240.8. From the new criteria, we conclude that Strategy 2 is the best, then Strategy 1, 3 and 4. In fact, Strategy 2 ensures the smaller mean loss and the individual extreme loss.

Definition. (CVaRMD). Let $\Pi_{T}$ be the profit of an investment and $q^{\alpha}\left(\Pi_{T}\right)$ be its $\alpha$-quantile. Denote $\Pi_{T^{\prime}}$ as the random loss more than $q^{\alpha}\left(\Pi_{T}\right)$, andmis the median of $\Pi_{T^{\prime}}$. CVaRMD in this paper is defined by

$$
C V a R M D^{\alpha}=E\left(\Pi_{T^{\prime}}+\left|\Pi_{T^{\prime}}-m\right|\right) .
$$

In Eq.(8), the first step of calculating CVaRMD is to determine the Cumulative Distribution Function (CDF) or the Probability Density Function (PDF)of $\Pi_{T^{\prime}}$.

Lemma. $\operatorname{Let} F_{\Pi_{T}}(y)$ be the $\mathrm{CDF}$ of $\Pi_{T}$. Then the PDF of $T_{T^{\prime}}$ is given by

$$
f_{\Pi_{T^{\prime}}}(y)=\left\{\begin{array}{cl}
\frac{f_{\Pi_{T}}(y)}{F_{\Pi_{T}}\left(q^{\alpha}\left(\Pi_{T}\right)\right)}, & y<q^{\alpha}\left(\Pi_{T}\right), \\
0, \quad y \geq q^{\alpha}\left(\Pi_{T}\right) .
\end{array}\right.
$$

Proof. The CDF of $\Pi_{T^{\prime}}$ is defined as follows:

$$
\begin{aligned}
F_{\Pi_{T^{\prime}}}(y)=P\left(\Pi_{T} \leq y \mid \Pi_{T}\right. & \left.\leq q^{\alpha}\left(\Pi_{T}\right)\right) \\
& =\frac{P\left(\Pi_{T} \leq y, \Pi_{T} \leq q^{\alpha}\left(\Pi_{T}\right)\right)}{P\left(\Pi_{T} \leq q^{\alpha}\left(\Pi_{T}\right)\right)} .
\end{aligned}
$$

When $y \geq q^{\alpha}\left(\Pi_{T}\right)$, then $F_{\Pi_{T^{\prime}}}(y)=1$; Otherwise, when $y<q^{\alpha}\left(\Pi_{T}\right)$, we have

$$
F_{\Pi_{T^{\prime}}}(y)=\frac{P\left(\Pi_{T} \leq y\right)}{P\left(\Pi_{T} \leq q^{\alpha}\left(\Pi_{T}\right)\right)}=\frac{F_{\Pi_{T}}(y)}{F_{\Pi_{T}}\left(q^{\alpha}\left(\Pi_{T}\right)\right)} .
$$

Therefore, by taking the derivative of $F_{\Pi_{T^{\prime}}}(y)$, the PDF of $\Pi_{T^{\prime}}$ is expressed by Eq.(4).

\subsection{The optimal hedging model}

When the firm purchases the oil options to hedge the extreme loss risk, the optimal hedging problem based on CVaRMD is expressed in Problem $\left(P_{1}\right)$ : 


\section{Crude Oil Options Hedging Based on a New Extreme Risk Measure}

where $\Pi_{T}^{p}$ is described in Eq.(1) $\operatorname{and} E\left(S_{T}\right)=\bar{S}$.

$$
\left(P_{1 .}\right)\left\{\begin{array}{l}
\min _{Z_{p}, K_{p}} \operatorname{CVaRM} M^{\alpha}\left(\Pi_{T}^{p}\right) \\
\text { s.t. } C=p_{0} \bar{S} Z_{p}
\end{array}\right.
$$

\subsection{Representations of the hedged portfolio based on copula functions}

This section aims to determine the probability distributions of $\Pi_{T}$ based on the derivations of famous Sklar's Theorem. Then, we express the CDF of the hedged portfolio in form of analysis.

Theorem. Suppose that a firm buys commodity options for hedging the import risk. If the call option expires ITM, then the cumulative distribution function of $\Pi_{T}^{p}$ is

$F_{\Pi_{T}^{p}}^{p}(u)=1-\int_{0}^{1}\left[\frac{\partial F_{P_{T} S_{T}}\left(\frac{u-F_{P_{0} S_{0}-\left(K_{p}+p_{0}\right) S_{T} Z_{p}}^{-1}}{z_{p}-1}\right)}{\partial w}-D_{1} C_{S_{T}, S_{T} P_{T}}(1-\right.$

$\left.\left.w, F_{S_{T} P_{T}}\left(\frac{u-F_{P_{0} S_{0}-\left(K_{p}+p_{0}\right) S_{T} Z_{p}}^{-1}(w)}{z_{p}-1}\right)\right)\right] d w$

where $F_{P_{0} S_{0}-\left(K_{p}+p_{0}\right) S_{T} Z_{p}}(t)=1-F_{S_{T}}\left(\frac{P_{0} S_{0}-t}{\left(K_{p}+p_{0}\right) Z_{p}}\right)$,

$F_{\left(Z_{p}-1\right) P_{T} S_{T}}(x)=1-F_{S_{T} P_{T}}\left(\frac{x}{z_{p}-1}\right)$.

If the commodity option is worthless, then the cumulative distribution function of $\Pi_{T}^{p}$ is

$$
F_{\Pi_{T}^{p}}(u)=1-\int_{0}^{1}\left[\frac{\partial v}{\partial w}-D_{1} C_{S_{T}, S_{T} P_{T}}(1-w, v)\right] d w,
$$

where $v=F_{S_{T} P_{T}}\left(F_{P_{0} S_{0}-p_{0} S_{T} Z_{p}}^{-1}(w)-u\right), F_{P_{0} S_{0}-p_{0} S_{T} z_{p}}(t)=1-F_{S_{T}}\left(\frac{P_{0} S_{0}-t}{p_{0} Z_{p}}\right)$ and $F_{-S_{T} P_{T}}(x)=1-F_{S_{T} P_{T}}(-x)$.

Proof. If the call option expires ITM, the gain of the hedged portfolio is

$$
\Pi_{T}^{p}=P_{0} S_{0}-\left(K_{p}+p_{0}\right) S_{T} Z_{p}+\left(Z_{p}-1\right) P_{T} S_{T} .
$$

In this case, we have

$$
\begin{gathered}
F_{\Pi_{T}^{p}}(u)=\int_{0}^{1} D_{1} C_{P_{0} S_{0}-\left(K_{p}+p_{0}\right) S_{T} Z_{p},\left(Z_{p}-1\right) P_{T} S_{T}}\left(w, F_{\left(Z_{p}-1\right) P_{T} S_{T}}(u\right. \\
\left.\left.-F_{P_{0} S_{0}-\left(K_{p}+p_{0}\right) S_{T} Z_{p}}(w)\right)\right) d w .
\end{gathered}
$$

Since $P_{0} S_{0}-\left(K_{p}+p_{0}\right) S_{T} Z_{p}$ and $\left(Z_{p}-1\right) P_{T} S_{T}$ decrease with regard to $S_{T}$ and $P_{T} S_{T}$, then the distributional function of $\Pi_{T}^{p}$ is 
Xing Yu, Weiguo Zhang, Yongjun Liu

$$
\begin{aligned}
\int_{0}^{1} D_{1}\left[w+F_{\left(Z_{p}-1\right) P_{T} S_{T}}\left(u-F_{P_{0} S_{0}-\left(K_{p}+p_{0}\right) S_{T} z_{p}}^{-1}(w)\right)-1\right. \\
+C_{S_{T}, S_{T} P_{T}}(1-w, 1 \\
\left.\left.-F_{\left(Z_{p}-1\right) P_{T} S_{T}}\left(u-F_{P_{0} S_{0}-\left(K_{p}+p_{0}\right) S_{T} Z_{p}}(w)\right)\right)\right] d w
\end{aligned}
$$

On the other hand, the probability distribution function of $P_{0} S_{0}-\left(K_{p}+p_{0}\right) S_{T} Z_{p}$ is

$$
F_{P_{0} S_{0}-\left(K_{p}+p_{0}\right) S_{T} Z_{p}}(t)=1-F_{S_{T}}\left(\frac{P_{0} S_{0}-t}{\left(K_{p}+p_{0}\right) Z_{p}}\right) .
$$

Similarly, the distributional function of $\left(Z_{p}-1\right) P_{T} S_{T}$ is

$$
F_{\left(Z_{p}-1\right) P_{T} S_{T}}(x)=1-F_{S_{T} P_{T}}\left(\frac{x}{z_{p}-1}\right) \text {. }
$$

From Eqs.(10) and (11), we obtain the cumulative distribution function when the call option expires ITM. When the pay-off of the call option at maturity is equal to 0 , the gain is expressed by $\Pi_{T}^{p}=P_{0} S_{0}-p_{0} Z_{p} S_{T}-S_{T} P_{T}$.

Since $F_{P_{0} S_{0}-p_{0} S_{T} z_{p}}(t)=1-F_{S_{T}}\left(\frac{P_{0} S_{0}-t}{p_{0} z_{p}}\right)$, and $F_{-S_{T} P_{T}}(x)=1-F_{S_{T} P_{T}}(-x)$, we thus have the distribution functions of $P_{0} S_{0}-p_{0} Z_{p} S_{T}$ and $-S_{T} P_{T}$. When the option is out of the money, the cumulative distribution function is

$$
F_{\Pi_{T}^{p}}(u)=\int_{0}^{1} D_{1} C_{P_{0} S_{0}-p_{0} S_{T} z_{p},-S_{T} P_{T}}\left(w, F_{-S_{T} P_{T}}\left(u-F_{P_{0} S_{0}-p_{0} S_{T} Z_{p}}^{-1}(w)\right)\right) d w .
$$

Due to the distributional functions of $P_{0} S_{0}-p_{0} Z_{p} S_{T}$ and $-S_{T} P_{T}$, the cumulative distribution function of $\Pi_{T}^{p}$ is shown in the Theorem.

\section{Empirical studies and comparatively analysis}

In this section, we provide an empirical study to illustrate the ideas of our proposed model and show the advantages of CVaRMD over CVaR in reducing the extreme risk. Based on the data collected from Database, we first model the marginal distributions and the dependence structure between the data series. Depending on the given computational procedure, we calculate the cumulative distribution function of the combined gain or loss of the hedged position at time $T$. Using the entire distribution function, we further compute the values of CVaR and CVaRMD. Then, the optimal strategies that minimize the extreme risk are derived. Finally, we assess the performance of the options hedging model and the different risk measures.

\subsection{Data, the marginal distributions and dependence structure}

The domestic enterprises in China, especially the refinery enterprises are importing more crude oil under the trend of the crude oil imports increasing in the foreign markets. We assume that a Chinese firm imports a unit crude oil over the next week. To pursue the abundant amount of available data, our data include weekly Brent crude oil price and the exchange rate(CNY/USD). The data are 


\section{Crude Oil Options Hedging Based on a New Extreme Risk Measure}

selected from July 8, 2011 to October 28, 2016 and the data sources are from Wind database. At initial time, the Brent crude oil price and the exchange rate $\operatorname{are} P_{0}=$ 45.6and $S_{0}=6.754$. We express rates of the crude oil price and the exchange rate by their logarithm of $r_{p}=\ln \left(P_{T}\right)-\ln \left(P_{0}\right) \quad$ and $r_{s}=\ln \left(S_{T}\right)-\ln \left(S_{0}\right)$, where $P_{T}$ and $S_{T}$ are the crude oil price and the exchange rate at time $T$. The weekly log-returns of the crude oil price and the exchange rate are shown in Fig.1.

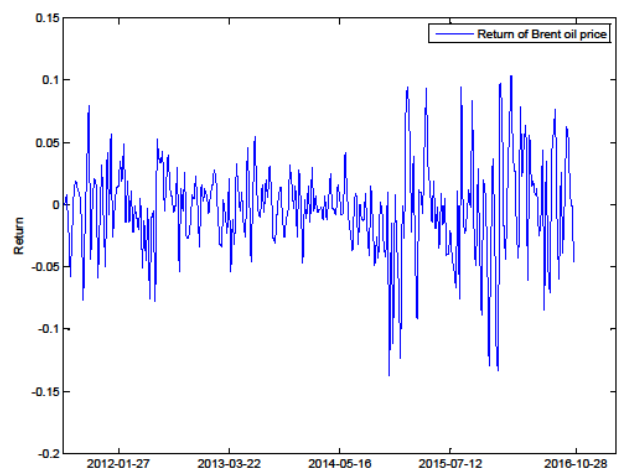

(a) Returns of the crude oil price

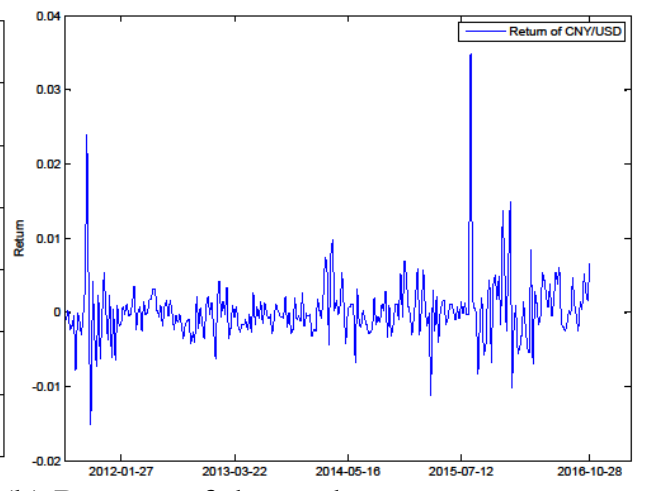

(b) Returns of the exchange rate

Fig. 1. Weekly returns of the crude price and the exchange rate CNY/USD

From Fig.1, we can find that returns of the crude oil price and the exchange rate show obvious heteroscedasticity, volatility, aggregation and sustainability. Then, $\mathrm{ARCH}$ effects and serial correlation of the returns are tested. The test results are presented in Table 2.

Table 2. Descriptive statistics of the crude oil price and the exchange rate

\begin{tabular}{ccc|ccc}
\hline Statistics & crude oil price & CNY/USD & Statistics & crude oil price & CNY/USD \\
\hline Mean & -0.0087 & 0.0002 & Skewness & -0.2031 & 0.9298 \\
max & 0.1752 & 0.0215 & Kurtosis & 3.0029 & 4.3297 \\
min & 0.2055 & 0.0133 & P-value & 0.500 & 0.0063 \\
Std.Dev & 0.0832 & 0.0074 & JB-statistic & 0.4952 & 15.6773 \\
\hline
\end{tabular}

Table 2 exhibits the descriptive statistics of the log-returns. Crude oil price shows higher volatility, as explained by the standard deviation. The most important inference from the descriptive analysis is that the null of Jacque Berra (JB) test is rejected. The results indicate that the return of the crude oil price is normally distributed. While the exchange rate return is not normally distributed. Therefore, we use GARCH models to examine the ARCH effect. ARCH-LM test shows that the crude oil price is a stationary stochastic process, but has heteroskedasticity. Our marginal models are built on the classical GARCH and GJR models. Model 
Xing Yu, Weiguo Zhang, Yongjun Liu

parameters are estimated by using the MLE method. Statistical values of the Akaike information criterion (AIC) and Bayesian information criterion (BIC) are reported in Table 3.

Table 3. Values of AIC and BIC criterion of GARCH models

\begin{tabular}{|c|c|c|c|c|c|c|c|}
\hline & Model & AIC & BIC & & Model & AIC & BIC \\
\hline $\mathrm{O}$ & GARCH-n & -158.6302 & 4207 & CNY/ & GARCH-n & -497.2833 & -478.0737 \\
\hline pri & GAF & -155.4 & 9 & USD & GA & 1 & -478 \\
\hline & & & & & & & -470.2630 \\
\hline & GJR-t & -154.6951 & -116.2760 & & GJR-t & -502.0352 & -463.6161 \\
\hline
\end{tabular}

Based on the criterions of AIC and BIC of the aforementioned GARCH models, from Table 3, we can find that, respectively, the crude oil price and the exchange rate are well fitted by GARCH-n and GARCH-t models. LM(K) statistic indicates that ARCH effects are likely to be found in both returns of the crude oil price and the exchange rate. Before proceeding with the evaluation of the hedging model, we estimate the parameters of the GARCH models. Tables 4 demonstrates the maximum likelihood results of GARCH-n and GARCH-t models.

\begin{tabular}{c|cc|cc}
\multicolumn{2}{c|}{ Table 4.Estimated parameter values of GARCH-n and GARCH-t models } \\
\hline Models & \multicolumn{2}{|c|}{ GARCH-n } & \multicolumn{2}{c}{ GARCH-t } \\
\hline Assets & \multicolumn{2}{|c|}{ Brent oil price } & \multicolumn{2}{c}{ Exchange rate } \\
\hline Parameter & Value & Std & Value & Std \\
C & -0.0057 & 0.0100 & $-8.1766 \mathrm{e}-04$ & $7.4826 \mathrm{e}-04$ \\
K & $4.1553 \mathrm{e}-04$ & $5.2288 \mathrm{e}-04$ & $2.7022 \mathrm{e}-06$ & $6.4787 \mathrm{e}-06$ \\
GARCH & 0.6804 & 0.1362 & 0.8552 & 0.2292 \\
ARCH & 0.2785 & 0.1591 & 0.1117 & 0.1769 \\
DOF & & & 4.6223 & 2.4450 \\
LLF & 82.8151 & & 257.8801 & \\
\hline
\end{tabular}

In what follows, we determine the dependence structure between the crude oil price and the exchange rate. Then, we select the best fitted copula function based on AIC and BIC criterions. Values of AIC and BIC are given in Table 5.

Table 5. AIC and BIC criterions of copula models

\begin{tabular}{llllll}
\hline Criterion & Gaussian-n & Student-t & Frank & Clayton & Gumbel \\
\hline AIC & 0.3770 & 0.3241 & 0.3137 & 2 & 2.13 \\
BIC & 2.6537 & 2.6008 & 2.5904 & 4.2767 & 4.4710 \\
\hline
\end{tabular}

From Table 5, we can find that according to the AIC and BIC values of all kinds of copulas, the Frank copula is the best fitting function to describe the dependence structure of the bivariate return series of the crude oil price and the exchange rate.

\subsection{Application of the options hedging models}

In this section, we apply the presented hedging model to a hypothetical firm wishing to hedge a unit Brent oil for a period of a week. The firm desires to hedge 


\section{Crude Oil Options Hedging Based on a New Extreme Risk Measure}

the oil price risk using number of $Z_{p}$ of call options. We assume that the firm allots a budget amount of $c$, which is equal to $2 \%, 6 \%, 11 \%$ and $16 \%$ of the naked position. According to the optimal hedging model and its solution steps, we obtain the optimal position of oil options and the values of CVaR and CVaRMD. Fig.2 expresses the results at the $95 \%$ confidence level of the hedged position.

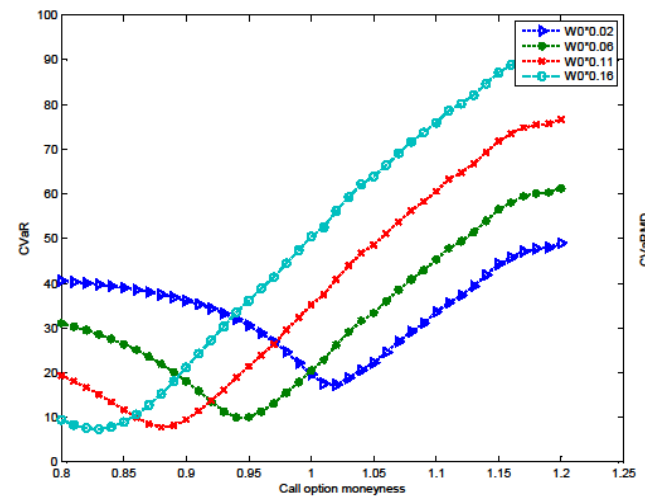

(a)CVaR with regard to call moneyness

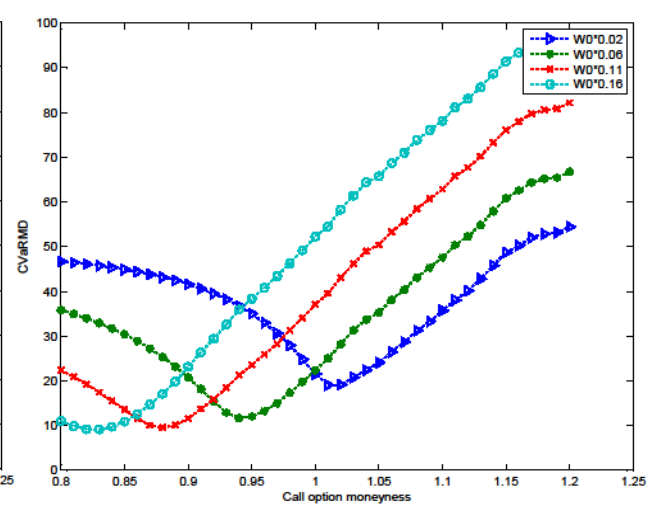

(b) CVaRMD with regard to call moneyness

Fig 2. Sensitivity of call moneyness

Fig.2 depicts the sensitivities of the call put moneyness (i.e. the ratio between the strike price and the current price of Brent oil price). Horizontal axis expresses the percentage of the spot position. The optimal strike price with larger budget is smaller. The call option with smaller strike price corresponds to a higher premium. The result is not surprising because more budget can support the firm to buy more options for hedging. For the other side, if the firm does not use oil options for hedging, its budget on buying option is 0 . Since we find that the more the budget is, the less the risk is, it is unwise for the firm not to buy oil options for hedging against the loss risk. We also find that the optimal strike price which minimizes CVaR or CVaRMD of the hedged portfolio is affected by the amount of cash spent on the hedging. This result confirms with Bajo et al.(2014).

The previous conclusions are upon the confidence level of 0.05 . While different decision makers have different confidence levels. We then study how the risk preferences of the firm affect the hedging effect. In Table 6, the budget is accounted for $1 \%$ of current value.

Table 6.CVaR and CVaRMD under different criterion

\begin{tabular}{ccc|ccc}
\hline$\alpha$ & CVaR & CVaRMD & $\alpha$ & CVaR & CVaRMD \\
\hline 0.01 & 11.1712 & 12.4463 & 0.025 & 9.0986 & 10.6875 \\
0.05 & 7.3424 & 9.1313 & 0.1 & 5.3911 & 7.3424 \\
\hline
\end{tabular}


Xing Yu, Weiguo Zhang, Yongjun Liu

In Table 6, we see that CVaR and CVaRMD decrease with regard to the confidence level. The results are consistent with the reality. When the hedger's risk aversion is larger, she/he cares the loss in a greater scope. Then the mean loss becomes smaller. In another aspect, hedgers with larger risk aversion are recommended to buy options for hedging.

\subsection{Discussion on the new risk measure}

Turning to another core of our empirical analysis, we evaluate the different hedging criterion of $\mathrm{CVaR}$ and CVaRMD. From the definitions of CVaR and CVaRMD, we can conclude that CVaR captures the mean loss while neglects the individual extreme loss. However, $\mathrm{CVaR}$ proposed in this paper traces both the mean and the individual extreme loss. To examine the robustness of the new risk measure in reducing the extreme risk, we simulate 200 possible future situations and compute the skewness values of the extreme loss under the strategies of minimizing CVaR or CVaRMD. The comparison results are shown in Fig.3.

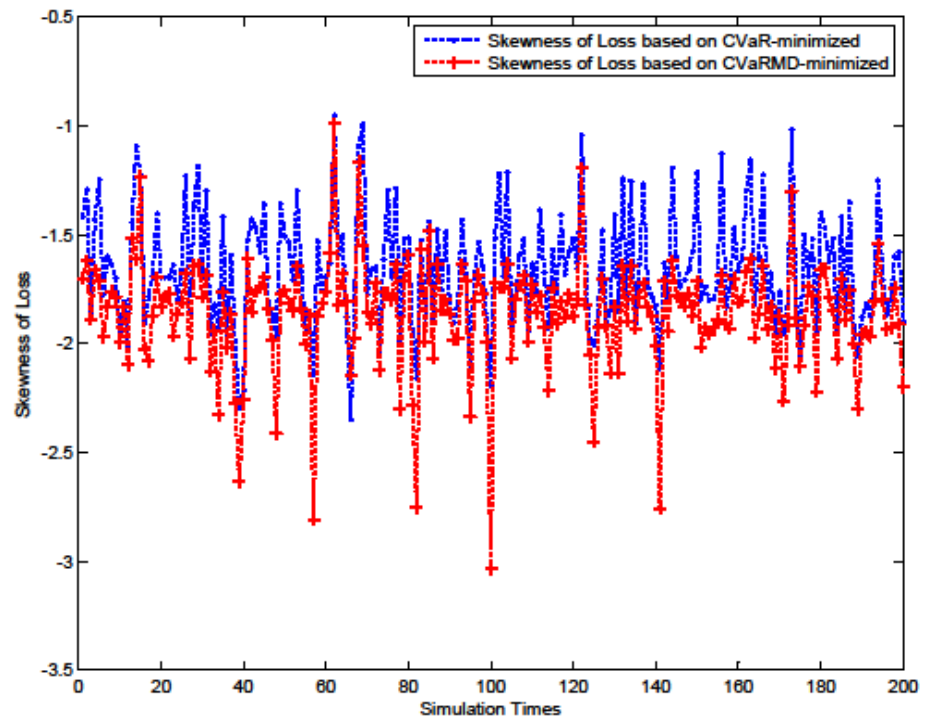

Fig.3 Skewness of the extreme loss on minimizing CVaR or CVaRMD

From Fig.3, we find that the negative skewness of CVaRMD minimizing criterion is remarkably larger than CVaR minimizing criterion. We know that a sequence with a larger negative skewness shows larger left deviation degree. In particular, when the return of an investment has a larger negative skewness, it is favorable toward investors.

To further identify the superiority of CVaRMD to CVaR, we first compare the variances under the two strategies that minimizing CVaRMD or CVaR. We use the Kruskal-Wallis test to present the comparison results. Here, the Kruskal-Wallis test is a nonparametric version of classical one-way ANOVA, and an extension of the 


\section{Crude Oil Options Hedging Based on a New Extreme Risk Measure}

Wilcoxon rank sum test to more than two groups. In this part, we refer to the routine of Bensoussan et al. (2014), who aimed to determine how various aspects weigh in the users' trust in the cloud computing technology using the KruskalWallis test. With the Kruskal-Wallis test statistic, based on the sum of ranks for each of the samples, if the medians of two or more categories of respondents differ in each of the first-level hypothesis formulated at 0.05 level of significance, thus resulting in the following second-level hypotheses:

H0: the groups' medians are all equal in each of the first-level hypothesis

$\mathrm{H} 1$ : at least one median is different in each of the first-level hypothesis

The output matrix $c$ of the pairwise comparison results from a multiple comparison test using the information contained in the stats structure. And we

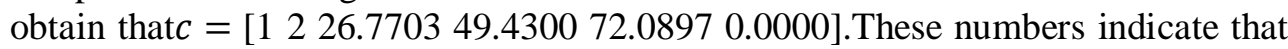
the mean of group 1 minus the mean of group 2 is estimated to be 49.4300, and at $95 \%$ confidence interval for the true difference of the means is [26.7703, 72.0897]. The $\mathrm{p}$-value for the corresponding hypothesis test that the difference of the means of groups 1 and 2 is significantly different from zero is 0.0000 . We also find that the confidence interval does not contain 0 , so the difference is significant at the $5 \%$ significance level. The p-value of 0.0000 also indicates that the difference of the means of groups 1 and 2 is significantly different from 0 . The ANOVA table provides additional test results, and the box plot in Fig.4 visually presents the summary statistics for each column.

Table 7.Kruskal-Wallis ANOVA

\begin{tabular}{llllll}
\hline Source & SS & df & MS & Chi-sq & Prob>Chi-sq \\
\hline Columns & 244332.5 & 1 & 244332.5 & 18.28 & $1.90771 \mathrm{e}-05$ \\
Error & 5088956.5 & 398 & 12786.3 & & \\
Total & 5333289 & 399 & & & \\
\hline
\end{tabular}

The returned value of pindicates that kruskalwallis rejects the null hypothesis that the two skewness values of the extreme loss come from the same distribution at a $1 \%$ significance level. For more intuitive comparison on CVaR and CVaRMD, we present the Kernel Cumulative Distribution Functions of the skewness values of the extreme loss in Fig.5: 
Xing Yu, Weiguo Zhang, Yongjun Liu

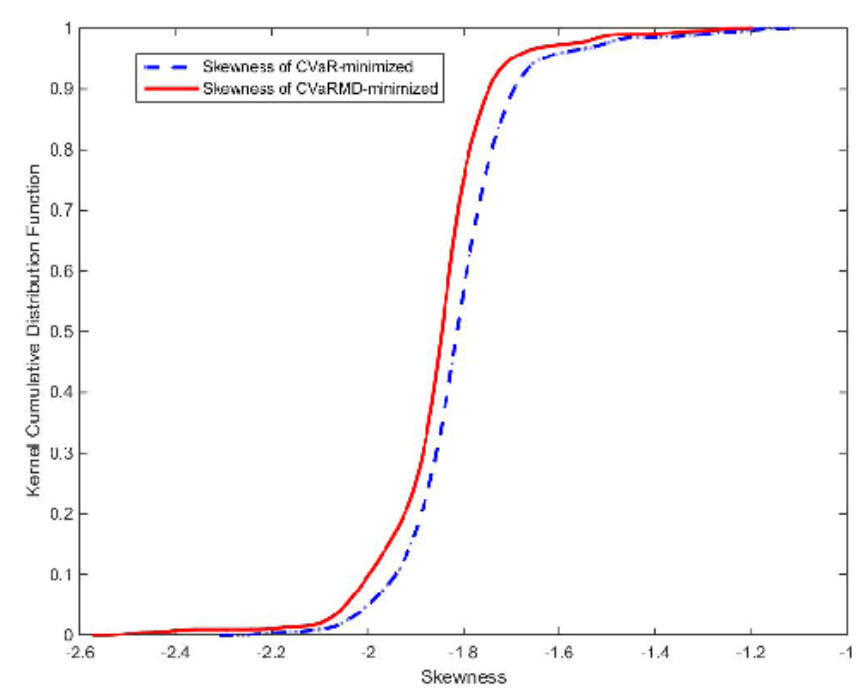

Fig 5. Kernel Cumulative Distribution Function of the skewness

If we compare the performance of the two risk measures by the skewness values of the extreme loss based on the Kernel Cumulative Distribution Functions, we should choose the strategy whose distribution function lies above the other because the probability of getting a larger negative skewness is larger for the strategy with a stochastically smaller distribution function. This method of comparison is common in finance and it is also known in probability theory as the first order stochastic dominate (Bodnar (2015)). From Fig.5, we observe that the criterion of CVaRMD over-performs CVaR. The probability of larger negative skewness values of the extreme loss is larger. To summarize, if the firm makes hedging strategy according to the criterion that minimizing CVaRMD, the extreme risk including the mean and the individual risk are relatively small.

\section{Conclusions}

In this paper, we examine the optimal crude oil hedging problem under the uncertainties of oil price and the exchange rate. The contributions of this paper is twofold to the existing studies. First, the dependence structure between oil price and the exchange rate is modeled through a copula function. And we represent the cumulative distributions of the combined gain or loss on the hedged position using copula functions. To do this, the options hedging model can be solved efficiently. Second, we propose a new risk measure called CVaRMD which is based on the integrated information of $\mathrm{CVaR}$ and the median deviation of the extreme loss. And the new risk measure incorporates the tail information including tail mean loss and tail extreme loss. According to the strategy of minimizing CVaRMD, the loss has a greater negative skewness, which is beneficial to the firm to hedge against the mean and individual extreme loss. 


\section{Crude Oil Options Hedging Based on a New Extreme Risk Measure}

Specifically, we apply Copula-GARCH method to estimate CVaR and CVaRMD of the hedged portfolio. Firstly, we employ GARCH and GJR process allowing for different error distributions of standard normal or students-t to model the marginals. Based on AIC and BIC criterions, we find that GARCH-n marginal distribution is best-fitted for the Brent oil price and GARCH-t model is best to fit the time series of the exchange rate. Frank copula describes the dependence structure of the return series quite well. For the two risk measures of CVaR and CVaRMD incorporated in this paper, we study sensitivities of the strike price and budge. It finds that crude oil options hedging helps the firm to hedge the oil importing risk. The optimal strike price is affected by the amount of cash spent on the hedging. But the optimal strike price of oil option decreases with respect to the budget. To illustrate the advantages of the proposed risk measure, we simulate future scenarios of the returns. Through comparison analysis, we find that the extreme loss from CVaRMD is more left skew than the ones from CVaR. Therefore, CVaRMD is superior to CVaR in controlling the extreme loss risk.

Copula-GARCH methodology is quite flexible to model the dependence between two assets or risk factors. One can further use different marginal distributions. As to the selection of a specific marginal distributions, more studies on the volatility modelling are needed.

\section{ACKNOWLEDGMENTS}

This paper is supported by Funds for International Cooperation and Exchange of the National Natural Science Foundation of China (71720107002), National Natural Science Foundation of China (No.71501076), Natural Science Fund of Guangdong (No.2014A030310454), Financial Service Innovation and Risk Management Research Base of Guangzhou, Social Science Foundation of Hubei Province of China(No.2018116),Humanities and Social Science Planning Fund from Ministry of Education(Grant No. 16YJAZH078).

\section{REFERENCES}

[1]Ahn,D.H., Boudoukh,J., Richardson,M., Whitelaw,R.F. (1999), Optimal Risk Management Using Options. The Journal of Finance, 54(1), 359-375; [2]Bajo,E., Barbi,M., Romagnobi, S. (2015), A Generalized Approach to Optimal Hedging with Option Contracts. The European Journal of Finance, 21(9), 713-733;

[3] Bodnar, T., Parolya,N., Schmid,W. (2015), On the Exact Solution of the Multi-Period Portfolio Choice Problem for an Exponential Utility under Return Predictability. European Journal of Operational Research, 246, 528-542; [4]Brahmasrene, T., Huang, J .C.,Sissoko, Y. (2014),Crude Oil Prices and Exchange Rates: Causality, Variance Decomposition and Impulse Response. Energy Economics, 44(2), 407-412; 
Xing Yu, Weiguo Zhang, Yongjun Liu

[5]Brayek,A. B., Sebai,S., Naoui,K. (2015), A Study of the Interactive Relationship between Oil Price and Exchange Rate: A Copula Approach and a DCC-MGARCH Model. Journal of Economic Asymmetries, 12(2):173-189; [6]Chen, H. T., Liu,L., Wang, Y .D., Zhu ,Y .M. (2016), Oil Price Shocks and U.S. dollar Exchange Rates. Energy, 112, 1036-1048;

[7]Chen,Y.H., Tu,A.H. (2013),Estimating Hedged Portfolio Value-At-Risk Using the Conditional Copula: An Illustration of Model Risk. International Review of Economics \& Finance, 27, 514-528;

[8]Deng, L., Ma, C .Q., Yang, W. (2011),Portfolio Optimization via pair CopulaGARCH-EVT-CVaR Model. Systems Engineering Procedia, 2, 171-181;

[9]Dinică̆,M.C., Balea,E. C. (2014),Natural Gas Price Volatility and Optimal Hedge Ratios. Economic Computation \& Economic Cybernetics Studies and Research;ASE Publishing, 48 (3):163-179;

[10]Domino, K., Blachowicz,T. (2015),The Use of Copula Functions for Modeling the Risk of Investment in Shares Traded on the Warsaw Stock Exchange. Physica A: Statistical Mechanics \& Its Application, 424(11), 142-151; [11]Ghosh,S. (2011), Examining Crude Oil Price-Exchange Rate Nexus for India during the Period of Extreme Oil Price Volatility. Applied Energy, 88(5), 1886-1889;

[12]Ito,T., Koibuchi,S., Sato,K., Shimizu,J. (2013),Exchange Rate Exposure and Risk Management: The Case of Japanese Exporting Firms. Journal of the Japanese and International Economies, 41, 17-29;

[13]Melnikov, A., Smirnov, I. (2012), Dynamic Hedging of Conditional Valueat-Risk. Insurance Mathematics \& Economics, 51(1), 182-190;

[14]Rivieccio,G., Luca,G.D. (2016), Copula Function Approaches for the Analysis of Serial and Cross Dependence in Stock Returns. Finance Research Letters, 17, 55-61;

[15]Salisu,A.A., Mobolaji,H. (2013),Modeling Returns and Volatility

Transmission between Oil Price and US-Nigeria Exchange Rate. Energy

Economics, 39(3), 169-176;

[16]Wong,K.P. (2003),Currency Hedging with Options and Futures. European Economic Review, 47(5), 833-839;

[17]Wong,K.P., Ming,C.(2009),Estimation of Multiple Period Expected Shortfall and Median Shortfall for Risk Management. Quantitative Finance, 12(5), 739-

754 ;

[18]Zhang,B., Wei,Y., Yu J, Lai,X., Peng,Z.(2014), Forecasting VaR and ES of Stock Index Portfolio: A Vine Copula Method. Physica A Statistical Mechanics \& Its Application, 416, 112-124. 